\title{
Type-2 Fuzzy Point
}

\author{
Mohammed Salih Mahdy Hussan, Munir Abdul Khalik Al-Khafaji \\ Department of Mathematics, College of Education, AL-Mustinsiryah University, Baghdad, Iraq \\ Email: mssm_1975@yahoo.com,mnraziz@yahoo.com
}

How to cite this paper: Hussan, M.S.M. and Al-Khafaji, M.A.K. (2019) Type-2 Fuzzy Point. Journal of Applied Mathematics and Physics, 7, 3067-3072. https://doi.org/10.4236/jamp.2019.712215

Received: June 11, 2019

Accepted: December 15, 2019

Published: December 18, 2019

Copyright $\odot 2019$ by author(s) and Scientific Research Publishing Inc. This work is licensed under the Creative Commons Attribution International License (CC BY 4.0).

http://creativecommons.org/licenses/by/4.0/

\begin{abstract}
The important role of the concept of type-2 fuzzy point in the formation of type-2 fuzzy open sets such as type-2 fuzzy $\tilde{\tilde{\delta}}$-closed set this important role make the main objective of this paper is to introduce the concept type-2 fuzzy point of type-2 fuzzy set an important definitions in the composition of this concept as $\tilde{\tilde{\alpha}}$-plane and the support of type-2 fuzzy set after preliminaries we present the definition of type-1 fuzzy set (fuzzy set) and fuzzy point and the special concepts that helped to configure them as support.
\end{abstract}

\section{Keywords}

Type-2 Fuzzy Set, $\tilde{\tilde{\alpha}}$-Plane, Fuzzy Point, Type-1 Fuzzy Set, Type-2 Fuzzy Point

\section{Introduction}

The main concept of a type- 1 fuzzy set (fuzzy set) is introduced by Zadeh in 1965 [1]. In [2], he introduced the concept, namely, type-2 fuzzy set in 1973 to classify a fuzzy set of type-1 fuzzy set when dealing with type-2 fuzzy set. Many of the scientists then introduced the concepts of type-2 fuzzy set, in 1976 introducing Mizumoto and Tanaka some properties of type-2 fuzzy set [3] and how to find the operations of type-2 fuzzy sets using the extension principle, it is the same rule that it has established Zadeh in 1973. Scientists then introduced several important concepts of the type-2 fuzzy set that correspond to the main concepts of the type-1 fuzzy set. In [4], Liu introduced the $\tilde{\tilde{\alpha}}$-plane concept of type-2 fuzzy set corresponding to the concept $\tilde{\alpha}$-level of the type-1 fuzzy set and the concept of support type-2 fuzzy set was developed by Mendel [5] corresponding to the concept of support type-1 fuzzy set. Mendel then introduced a very important concept interval type-2 fuzzy sets [5]. The study of type-2 fuzzy sets was expanded to include the topology study of type- 1 fuzzy set with the introduction of fuzzy topology by Chang in 1968 [6]. Zhang in 2013 offered the 
interval type-2 fuzzy topological space [7] and then introduced Mohammad and Munir General type-2 fuzzy topological space [8]. The type-2 fuzzy open sets such as type-2 fuzzy $\tilde{\tilde{\delta}}$-preopen set and type-2 fuzzy $\tilde{\tilde{e}}$-open set, we cannot configure its own definition only after introduce type-2 fuzzy point concept that belongs to the general fuzzy type- 2 topological space.

\section{Preliminaries}

This section paves the way to introducing the concept of type-2 fuzzy point by providing definitions of special important concept type- 1 fuzzy set and type-2 fuzzy set after submitting the special definition to the clusters.

\subsection{Definition [1]}

If $X$ is a collection of objects with generic element $\chi$, then a fuzzy subset $\tilde{A}$ in $X$ is characterized by a membership function; $\tilde{\aleph}_{\tilde{A}}: X \rightarrow I$, where $I$ is the closed unit interval $[0,1]$, then we write a fuzzy set $\tilde{A}$ by the set of points: $\tilde{A}=\left\{\left(\chi, \tilde{\aleph}_{\tilde{A}}(\chi)\right) \mid \chi \in X, 0 \leq \tilde{\aleph}_{\tilde{A}}(\chi) \leq 1\right\}$.

\subsection{Definition [9]}

The support of a type-1 fuzzy set $\tilde{A}$ (denoted by $S(\tilde{A})$ ) which is the crisp set of all $\chi \in X,\left\{\chi: \tilde{\aleph}_{\tilde{A}}(\chi)>0\right\}$.

In order to represent an element of a type-1fuzzy set $\tilde{A}$, we provide a concept in type-1 fuzzy set theory that is a special case of $\tilde{\alpha}$-level sets, which is called fuzzy points used for inclusion of elements to fuzzy sets.

\subsection{Definition [10]}

A type-1 fuzzy point $\tilde{\wp}$ in a set $X$ is also a type-1 fuzzy set with membership function:

$$
\tilde{\aleph}_{\tilde{\mathfrak{g}}}(\chi)=\left\{\begin{array}{ll}
\kappa & \text { for } \chi=\gamma \\
0 & \text { for } \chi \neq \gamma
\end{array},\right.
$$

where $\chi \in X$ and $0<\kappa<1, \gamma$ is called the support of $\tilde{\wp}$ and $\kappa$ the value of $\tilde{\wp}$. We denote this type-1 fuzzy point by $\tilde{\wp}$. Two fuzzy points $\tilde{\wp}_{\chi_{\kappa}}$ and $\tilde{\wp}_{\gamma_{\kappa}}$ are said to be distinct if and only if $\chi \neq \gamma$. A type-1 fuzzy point $\tilde{\wp}$ is said to belong to a type-1 fuzzy subset $\tilde{A}$ in $X$, denoted by $\tilde{\wp} \in \tilde{A}$ if and only if $\kappa \leq \tilde{\aleph}_{\tilde{A}}(\chi)$.

\subsection{Definition [2] [5]}

Let $X$ be a finite and non-empty set, which is referred to as the universe a type-2 fuzzy set, denoted by $\tilde{\tilde{A}}$ is characterized by a type- 2 memberships function $\tilde{\tilde{\aleph}}_{\tilde{\tilde{A}}}(\chi, \tilde{u})$, as

$$
\tilde{\tilde{\aleph}}_{\tilde{A}}: X \times I \rightarrow I^{J_{\chi}}\left(J_{\chi} \subseteq I\right), I=[0,1],
$$

where $\chi \in X$ and $\tilde{u} \in J_{\chi}$, that is 
$\tilde{\tilde{A}}=\left\{\left((\chi, \tilde{u}), \tilde{\tilde{\aleph}}_{\tilde{\tilde{A}}}(\chi, \tilde{u})\right)\right.$ : where $\chi \in X$ and $\tilde{u} \in J_{\chi} \subseteq I$, where $\left.0 \leq \tilde{\tilde{\aleph}}_{\tilde{\tilde{A}}}(\chi, \tilde{u}) \leq 1\right\}$.

We can give a new wording to $\tilde{\tilde{A}}$

$$
\begin{aligned}
& \tilde{\tilde{A}}=\sum_{\chi \in X} \sum_{\tilde{u} \in v_{\chi}} \frac{\tilde{\tilde{\aleph}}_{\tilde{\tilde{A}}}(\chi, \tilde{u})}{(\chi, \tilde{u})} \\
& \tilde{\tilde{A}}=\sum_{\chi \in X} \sum_{\tilde{u} \in v_{\chi}} \frac{\frac{\tilde{\tilde{\mathcal{F}}}_{\chi}(\tilde{u})}{\tilde{u}}}{\chi}, v_{\chi} \subseteq[0,1]
\end{aligned}
$$

where $\tilde{\tilde{\mathcal{F}}}_{\chi}(\tilde{u})=\tilde{\tilde{\aleph}}_{\tilde{\tilde{A}}}(\chi, \tilde{u})$ and $\Sigma \Sigma$ denote the union in discrete sets and $\Sigma$ is replaced by $\int$ is continuous universes are set. The class of all type-2 fuzzy set of $\chi \in X \neq \varnothing$ denoted by $\tilde{\tilde{\mathbb{F}}}_{T-2}(X)$.

A type-2 fuzzy set universes set [3], denoted by, such that

$$
\tilde{\tilde{X}}=\sum_{\chi \in X} \sum_{\tilde{u} \in[1,1]} \frac{\frac{1}{\tilde{u}}}{\chi} .
$$

A type-2 fuzzy empty set [3], denoted by

$$
\tilde{\tilde{\varnothing}}=\sum_{\chi \in X} \sum_{\tilde{u} \in[0,0]} \frac{\frac{1}{\tilde{u}}}{\chi} .
$$

Interval type-2 fuzzy set [5], when all the $\underset{\tilde{\tilde{\aleph}}}{\tilde{\tilde{A}}}(\chi, \tilde{u})=1$, for all $\underset{\tilde{\tilde{B}}}{\tilde{B}} \chi \in X$.

The operations of type-2 fuzzy set [3], consider $\tilde{\tilde{A}}$ and $\tilde{\tilde{B}}$ are two type-2 fuzzy sets and the membership grades of $\tilde{\tilde{A}}$ and $\tilde{\tilde{B}}$ respectively, we can represented by

$$
\tilde{\tilde{\aleph}}_{\tilde{\tilde{A}}}(\chi)=\sum_{\tilde{u} \in v_{\chi}^{\tilde{u}}} \frac{\tilde{\mathcal{F}}_{\chi}(\tilde{u})}{\tilde{u}} \text { and } \tilde{\tilde{\aleph}}_{\tilde{\tilde{B}}}(\chi)=\sum_{\tilde{w} \in v_{\chi}^{\tilde{x}}} \frac{\tilde{\tilde{\mathcal{R}}}_{\chi}(\tilde{w})}{\tilde{w}},
$$

where $\tilde{\tilde{\mathcal{F}}}_{\chi}(\tilde{u}), \tilde{\tilde{\mathcal{R}}}_{\chi}(\tilde{w}) \in \mathfrak{T}=[0,1]$ and.

The union of two type-2 fuzzy sets is defined as

$$
\tilde{\tilde{A}} \cup \tilde{\tilde{B}} \Leftrightarrow \tilde{\tilde{\aleph}}_{\tilde{\tilde{A}} \cup \tilde{\tilde{B}}}(\chi)=\sum_{\tilde{u} \in U_{\chi}^{\tilde{u}}} \sum_{\tilde{w} \in U_{\chi}^{\tilde{w}}} \frac{\tilde{\tilde{\mathcal{F}}}_{\chi}(\tilde{u}) \tilde{\wedge} \tilde{\tilde{\mathcal{R}}}_{\chi}(\tilde{w})}{\tilde{u} \tilde{\vee} \tilde{w}} .
$$

The intersection of two type-2 fuzzy sets is defined as

$$
\tilde{\tilde{A}} \cap \tilde{\tilde{B}} \Leftrightarrow \tilde{\tilde{\aleph}}_{\tilde{\tilde{A}} \cap \tilde{\tilde{B}}}(\chi)=\sum_{\tilde{u} \in u_{\chi}^{\tilde{u}}} \sum_{\tilde{w} \in v_{\chi}^{\tilde{\mathcal{F}}}} \frac{\tilde{\tilde{\mathcal{F}}}_{\chi}(\tilde{u}) \tilde{\wedge} \tilde{\tilde{\mathcal{R}}}_{\chi}(\tilde{w})}{\tilde{u} \tilde{\wedge} \tilde{w}} .
$$

The containment type-2 fuzzy sets are defined as $\tilde{\tilde{A}} \subseteq \tilde{\tilde{B}} \Leftrightarrow \tilde{\tilde{A}} \cap \tilde{\tilde{B}}=\tilde{\tilde{A}}$.

The complement of type-2 fuzzy set defined as $(\neg \tilde{\tilde{A}})=\sum_{\tilde{u} \in v_{\chi}^{\tilde{u}}} \frac{\tilde{\tilde{\mathcal{F}}}_{\chi}(\tilde{u})}{1-\tilde{u}}$.

A normal type-1 fuzzy set $\tilde{A}$ is one for height equals 1 , otherwise it is called subnormal [9].

\subsection{Definition [3]}

A normal type-2 fuzzy set $\tilde{\tilde{A}}$ is one for which $\max _{\chi \in X} \tilde{\tilde{\aleph}}_{\tilde{\tilde{A}}}(\chi, \tilde{u})=1$. 


\subsection{Definition [5]}

The support of a type-2 fuzzy set $\tilde{\tilde{A}}$ denoted $S(\tilde{\tilde{A}})$ comprises all $(\chi, \tilde{u}) \in X \times I$ such that $\tilde{\tilde{\aleph}}_{\tilde{\tilde{A}}}(\chi, \tilde{u})>0$.

\subsection{Definition [4]}

The two damnation $\tilde{\tilde{\alpha}}$-plane, denoted $\tilde{\tilde{A}}_{\tilde{\tilde{\alpha}}}$ is the union of all primary membership whose secondary grades are greater than or equal special value $\tilde{\tilde{\alpha}}$ that is: $\tilde{\tilde{A}}_{\tilde{\alpha}}=\bigcup_{\chi \in X}\left\{(\chi, \tilde{u}): \tilde{\tilde{\aleph}}_{\tilde{\tilde{A}}}(\chi, \tilde{u}) \geq \tilde{\tilde{\alpha}}, \tilde{\tilde{\alpha}} \in[0,1]\right\}$.

\subsection{Definition [8]}

Let $\tilde{\tilde{T}}$ be the collection of type-2 fuzzy sets over $X \neq \varnothing$ then $\tilde{\tilde{T}}$ is called to be general type-2 fuzzy topology on $X \neq \varnothing$.

If

1) $\tilde{\tilde{X}}, \tilde{\tilde{\varnothing}} \in \tilde{\tilde{T}}$;

2) $\tilde{\tilde{A}} \cap \tilde{\tilde{B}} \in \tilde{\tilde{T}}$;

3) $\bigcup_{i \in N} \tilde{\tilde{A}} \in \tilde{\tilde{T}}$ for $\quad \tilde{\tilde{A}}_{i} \in \tilde{\tilde{T}}$.

The pair $(\tilde{\tilde{X}}, \tilde{\tilde{T}})$ is said to general type-2 fuzzy topological space over $X$ and the member of $\tilde{\tilde{T}}$ is said to be type-2 fuzzy $\tilde{\tilde{T}} \sim$ open sets in $X$ and type-2 fuzzy sets are said type-2 fuzzy $\tilde{\tilde{T}} \sim$ closed sets in X, if its complement $\neg \tilde{\tilde{A}} \in \tilde{\tilde{T}}$.

We must note that all the type- 2 fuzzy sets are normal type- 2 fuzzy sets so as to complete the topological construction and especially check identity law $(\tilde{\tilde{A}} \cap \tilde{\tilde{\varnothing}}=\tilde{\tilde{\varnothing}})$.

\section{Type-2 Fuzzy Point}

The introduction of this section is the end of which the paper developed by the special case of $\tilde{\tilde{\alpha}}$-plane, which claims type-2 fuzzy point.

\subsection{Definition}

A type-2 fuzzy point $\tilde{\tilde{\wp}}$ in a set $X$ is also a type- 2 fuzzy set with secondary membership function:

$$
\tilde{\tilde{\aleph}}_{\tilde{\tilde{\xi}}}(\chi, \tilde{u})=\left\{\begin{array}{ll}
\kappa & \text { for } \chi=\gamma \\
0 & \text { for } \chi \neq \gamma
\end{array},\right.
$$

where $\chi \in X$ and $0<\kappa<1, \gamma$ is called the support of $\tilde{\tilde{\wp}}$ and $\kappa$ the value of $\tilde{\tilde{\wp}}$.

\subsection{Example}

Let $X=\{a, b\}, \tilde{\tilde{A}}=\{((a, 0.5), 0.4),((b, 0.8), 0)\}$ is a type-2 fuzzy point

$$
S(\tilde{\tilde{A}})=\{(a, 0.5)\} \text { and we have } \tilde{\tilde{\aleph}}_{\tilde{\tilde{\xi}}}(\chi, \tilde{u})= \begin{cases}\kappa=0.4 & \chi=\gamma \\ 0 & \chi \neq \gamma\end{cases}
$$




$$
\begin{aligned}
& \Rightarrow \text { that is } \tilde{\tilde{\aleph}}_{\tilde{\tilde{\xi}}}(\chi, \tilde{u})=\tilde{\tilde{\aleph}}_{\tilde{\xi}}(a, 0.5)=\kappa=0.4 \\
& \tilde{\tilde{\aleph}}_{\tilde{\tilde{p}}}(\chi, \tilde{u})=\tilde{\tilde{\aleph}}_{\tilde{\tilde{\xi}}}(b, 0.8)=0
\end{aligned}
$$

We denote this a type-2 fuzzy point by $\tilde{\tilde{\wp}}$. Two a type-2 points $\tilde{\tilde{\wp}}_{\chi_{\kappa}}$ and $\tilde{\tilde{\wp}}_{\gamma_{\lambda}}$ are said to be distinct if and only if $\chi \neq \gamma$.

\subsection{Serious Results}

The means of determining belonging set or fuzzy point in the subject of the fuzzy set is to compare with the membership function, but in the second type-2 fuzzy set we use the containment property given by Mizumotoand Tanaka $\tilde{\tilde{A}} \subseteq \tilde{\tilde{B}} \Leftrightarrow \tilde{\tilde{A}} \cap \tilde{\tilde{B}}=\tilde{\tilde{A}}[3]$.

\subsection{Example}

Let

$$
\begin{gathered}
X=\left\{\chi_{1}, \chi_{2}, \chi_{3}\right\} \\
\tilde{\tilde{A}}=\left\{\left(\left(\chi_{1}, 0.7\right), 0.8\right),\left(\left(\chi_{1}, 0.6\right), 1\right),\left(\left(\chi_{2}, 0.4\right), 1\right),\left(\left(\chi_{3}, 0.8\right), 0.9\right),\left(\left(\chi_{3}, 0.5\right), 1\right)\right\} \\
\tilde{\tilde{\wp}}=\left\{\left(\left(\chi_{1}, 0.6\right), 0.8\right),\left(\left(\chi_{2}, 0.3\right), 0.8\right),\left(\left(\chi_{3}, 0.5\right), 0\right)\right\} \\
\tilde{\tilde{\wp}} \cap \tilde{\tilde{A}}\left(\chi_{1}\right)=\frac{0.8 \wedge 0.8}{0.7 \wedge 0.6}+\frac{1 \wedge 0.8}{0.6 \wedge 0.6} \\
\tilde{\tilde{\wp}} \cap \tilde{\tilde{A}}\left(\chi_{1}\right)=(0.6, \max (0.8,0.8))=\left(\left(\chi_{1}, 0.6\right), 0.8\right) \\
\tilde{\tilde{\wp}} \cap \tilde{\tilde{A}}\left(\chi_{2}\right)=\frac{1 \wedge 0.8}{0.4 \wedge 0.3} \Rightarrow \frac{0.8}{0.3}=\left(\left(\chi_{2}, 0.3\right), 0.8\right) \\
\tilde{\tilde{\wp}} \cap \tilde{\tilde{A}}\left(\chi_{3}\right)=\frac{0.9 \wedge 0}{0.8 \wedge 0.5}+\frac{1 \wedge 0}{0.5 \wedge 0.5} \\
\tilde{\tilde{\xi}} \cap \tilde{\tilde{A}}\left(\chi_{3}\right)=(0.5, \max (0,0))=\left(\left(\chi_{3}, 0.5\right), 0\right) \\
\Rightarrow \text { therefore } \tilde{\tilde{\xi}} \cap \tilde{\tilde{A}}=\tilde{\tilde{\xi}} \Leftrightarrow \tilde{\tilde{\xi} \subseteq \tilde{A}} .
\end{gathered}
$$

\section{Conclusion}

The important role played by the type- 2 fuzzy point in configuring continuous function after building new concepts from open type-2 fuzzy set made us offer this new concept. This concept allows for future dealings with the concept of neighborhood in general type-2 fuzzy topological space. In order for us to be able to configure such as type-2 fuzzy $\tilde{\tilde{\delta}}$-preopenset and type-2 fuzzy $\tilde{\tilde{e}}$-open set only and to examine the relationships among them.

\section{Acknowledgements}

Thanks and appreciation to all who contributed to the publication and composition of the main idea of the research and especially Prof. Jerry Mendel and Prof. Mohammad Reza Rajati. 


\section{Conflicts of Interest}

The authors declare no conflicts of interest regarding the publication of this paper.

\section{References}

[1] Zadeh, L.A. (1965) Fuzzy Sets. Information and Control, 8, 338-353. https://doi.org/10.1016/S0019-9958(65)90241-X

[2] Zadeh, L.A. (1975) The Concept of a Linguistic Variable and Its Application to Approximate Reasoning-1. Information Sciences, 8, 199-249. https://doi.org/10.1016/0020-0255(75)90036-5

[3] Mizumoto, M. and Tanaka, K. (1976) Some Properties of Fuzzy Sets of Type-2. Information and Control, 31, 312-340. https://doi.org/10.1016/S0019-9958(76)80011-3

[4] Liu, F.L. (2008) An Efficient Centroid Type-Reduction Strategy for General Type-2 Fuzzy Logic System. Information Sciences, 178, 2224-2236. https://doi.org/10.1016/j.ins.2007.11.014

[5] Mendel, J.M. (2001) Uncertain Rule-Based Fuzzy Logic Systems: Introduction and New Directions. Prentice-Hall, New Jersey.

[6] Chang, C.L. (1968) Fuzzy Topological Spaces. Journal of Mathematical Analysis and Applications, 24, 182-190. https://doi.org/10.1016/0022-247X(68)90057-7

[7] Zhang, Z.M. (2013) On Characterization of Generalized Interval Type-2 Fuzzy Rough Sets. Information Sciences, 219, 124-150. https://doi.org/10.1016/j.ins.2012.07.013

[8] Hussan, M.S.M. and AL-Khafaji, M.A.K. (2018) General Type-2 Fuzzy Topological Spaces, Advances in Pure Mathematics, 8, 771-781. https://doi.org/10.4236/apm.2018.89047

[9] Klir, G.J. and Yuan, B. (1995) Fuzzy Sets and Fuzzy Logic Theory and Application. Prentice Hall, New Jersey.

[10] Wong, C.K. (1974) Fuzzy Points and Local Properties of Fuzzy Topology. Journal of Mathematical Analysis and Applications, 46, 316-328.

https://doi.org/10.1016/0022-247X(74)90242-X 